\title{
PRIMARY USE CASE IMPLEMENTATION OF INTERNATIONAL PATIENT SUMMARY ON OpEnEHR PLATFORM
}

\author{
Evgeniy Krastev ${ }^{1}$, Petko Kovatchev ${ }^{1}$, Dimitar Tcharaktchiev ${ }^{2}$ and Simeon Abanos ${ }^{1}$ \\ ${ }^{I}$ Faculty of Mathematics and Informatics, Sofia University St. Kliment Ohridsky, Bulgaria \\ 5 James Bouchier blvd., 1164 Sofia, Bulgaria \\ ${ }^{2}$ Department of Medical Informatics, Medical University, Sofia, Bulgaria \\ University Hospital of Endocrinology, 2 Zdrave str., 1431 Sofia, Bulgaria
}

\begin{abstract}
The International Patient Summary (IPS) comprises the essential clinical data that must be available in case an European citizen needs unscheduled or urgent health care while abroad. The implementation of cross- border exchange of IPS is among the highest priorities of the European commission. Detailed functional requirements and use cases have been elaborated in addition to a new IPS standard in recent years as part of the efforts for enabling interoperability in information exchange and continuity of care. This paper presents a practicable approach and a solution to the problem of achieving semantic interoperability among the IPS sources in the European union. A novelty approach in this paper is first to design and analyze the business process of the primary use case and after that to propose a solution. It allows to identify several activities that are out of the scope in the existing documents and at the same time require special attention at the stage of software implementation. The proposed solution makes use of the openEHR information model to represent the IPS standard dataset in terms of archetypes. This way we preserve the semantic context in clinical concepts as well as introduce interoperability among data providers. Moreover, it overcomes the severe drawback of the message paradigm, where every two communicating parties must apply transformation of existing data that is specific to their needs. The discussion considers a computer experiment with real data employing a fully functional web application for IPS cross-border exchange.
\end{abstract}

\section{KEYWORDS}

International Patient Summary, Use Case, Business Process Model and Notation, openEHR Information Model, Web Application, Semantic Interoperability

\section{INTRODUCTION}

The free movement of people poses several challenges to continuity of care and coordination of activities in cases of health accidents or emergency situations. Essential clinical data is urgently needed in understandable form so that the patient receives timely and adequate medical therapy. Reliable information like the patient's history of past illnesses, allergies and intolerances is available in the country of permanent stay and quite often it is not accessible at the time of hospitalization of a foreign citizen. Prevention the spread of infectious diseases such as COVID-19 requires travelers to provide evidence about their immunization status. Knowledge about the past illnesses of a foreign employee is an important factor in preventing workplace accidents in executing duties incompatible with his health status. These are only few of the typical healthcare problems that people might encounter while abroad. The seriousness of the problems becomes obvious once we take in consideration the large number of tourists in addition to people who migrate seeking better employment in developed countries (Footman, et al., 2014).

The European commission realized long ago the need for establishing a standard for the patients' minimal dataset of health information that is necessary to ensure continuity and coordination in administering medical treatment activities. The European Patients- Smart Open Services (epSOS) project proposed a pilot infrastructure for sharing and exchanging patient summary records (European Commission, 2012). The interoperability of health information systems (HIS) has become an obligatory requirement in cross- border 
exchange of patient summary. It sparked the development of interoperable solutions based on best practices in implementing eHealth standards and specifications (European Commission, 2015). An important step in this direction is the specification of detailed functional requirements and use case specifications (European Commission, 2018) applicable to the current standard EN 17269:2019 for International Patient Summary (IPS) (CEN/TC 251, 2019a). Nowadays special attention is dedicated to standards and specifications that enable semantic interoperability in exchange of clinical data. It allows to use information models and technologies for preserving the semantic context in which clinical data has been generated and make it equally understandable in the language of the receiving country. Typical representatives of such standards and specifications correspondingly, EN 13606:2019 (ISO/TC 215, 2019b) and openEHR (openEHR, 2020a), make use of the Archetype information model (openEHR, 2020b).

The review of existing literature sources shows that important implementation issues are usually not taken in consideration. For example, there is no publication that attempts to create a business process model and analyze the activities in cross- border exchange of IPS according to the use cases outlined by the European Commission. The primary use case considers a peer- to- peer message exchange of IPS clinical data between two countries. At the same it is recognized that European countries employ different eHealth information models for representing IPS entities, while some of them are even incompatible with internationally approved standards and specifications. Therefore, these use case do not support direct connectivity between major healthcare information system in Europe and semantic interoperability or even interoperability in cross- border exchange of IPS remains a serious challenge in practice.

A novel approach is here proposed for the development of a solution to this problem based on the analysis of business process model. The business process model makes use of Business Process Model and Notation 2.0 (BPMN) (Freund \& Rücker, 2020). This model elaborates the primary case study with the objective of achieving semantic interoperability among all countries participating in IPS data exchange. A novelty from practical point of view is the web application we have developed on openEHR platform to demonstrate the execution activities (Gutiérrez, 2015). Both the business process model and the web application for IPS exchange have no analogs in public available sources. The here reported results are part of ongoing eHealth research program (Krastev, et al., 2019), (Tcharaktchiev, et al., 2020), (Krastev, et al., 2020).

The main results in this paper are presented in the following four sections. Section 2 formulates the problem in terms of functional requirements and participants in the primary use case. Section 3 presents a BPMN model of the primary use case and elaborates on the web application that implements the activities in that business process. Section 4 evaluates the results obtained from the computer experiments and Section 5 summarizes the advantages of the business process model for executing the primary use case and the potential applications of the web application that implements it.

\section{PROBLEM STATEMENT}

The current IPS standard (CEN/TC 251, 2019a) has been developed with the initial objective to support the use case of cross- border exchange of essential patient's clinical data in situations of unscheduled care. During its development process the scope of application has been extended to cover scenarios of both scheduled and unscheduled care that involve the patient while he is abroad or in the country of permanent stay (CEN/TC 251, 2018). For clarity in this paper we refer to the primary use case as the use case of IPS cross- border exchange abroad related to unscheduled or scheduled care.

The IPS standard determines a well- structured model of the clinical document and comprises 6 required sections, namely, Patient Attributes, Allergies and Intolerances, Medication Summary, Problems, Provenance and a conditional Cross- Border section. The conditional section is ignored in the secondary use cases dealing with scheduled and unscheduled care in the country, where the patient lives permanently. The first three IPS sections are mandatory, while the standard allows the list required sections to be extended with additional "required if- known" sections. This way the IPS standard can be accommodated for exchange of clinical data in other use cases like for example exchanging clinical data between registers for rare diseases (Tcharaktchiev, et al., 2020).

The official definition of the primary use case involves the following actors, where for uniformity we preserve in bold their original notations (European Commission, 2018) (European Commission, 2020): 
Human actors:

- Patient is the citizen from country A seeking health care service in country B. Although a Patient may have different IPS documents

- HCP B is the healthcare professional providing health care service in country B

\section{$\checkmark$ Institutional actor:}

- HCPO B is the healthcare service providers organization in country B. All healthcare professionals belong to a specific HCPO.

$\checkmark$ System actors:

- NCP A denotes the national contact point in country A. Respectively, NCP B denotes the national contact point in country B. The national contact point provides visibility to a single IPS copy to the outside world. Hence, a Patient could have at most one IPS document in each country.

For clarity of presentation we omit semantic and business rule services that are part of the complete description of the System actors.

A description of the flow of events in the primary use case is presented in relation to key functional requirements in (European Commission, 2018), where the communication between the above identified actors is visualized in a UML Sequence diagram. In short, a Patient needs health assistance in country $\mathbf{B}$, he visits HCP B from HCPO B and HCP B wants to display the patient's IPS that is maintained by NCP A. HCP B establishes connection with NCP A through NCP B, the Patient identity is confirmed and after that the Patient authorizes HCP B to extract his IPS from NCP A. Finally, HCP B displays the IPS document and executes the requested health assistance taking in consideration the clinical data.

Although this description sounds complete enough it appears to be very difficult to implement it in practice because it avoids answering several important questions. For simplicity, assume both national contact points implement the IPS standard. First, this use case considers data exchange between two countries that in general use different information models to represent the same IPS standard. Note, that the IPS standard does not impose constraints on the information model that could be used to implement its data structures. The primary use case leaves out of scope the event of transformation needed between these two different models. Moreover, if we extend the primary use case and assume that country A could be any other European country, then we find out more complications in the practical implementation of this use case. For example, then NCP B must invest a lot in support for transformation between the information model adopted in country $\mathbf{B}$ and any other information model employed by the other national contact points. Besides, most of the time such transformations are "one- way". Another important issue is how to preserve the semantic context in the original IPS document during its digital transformation. This issue underpins the importance of using information models that support semantic interoperability. However, experience in using such models shows that interoperability even between such information models is difficult to achieve (Krastev, et al., 2020). Finally, the primary use case assumes HCP B can display the IPS. It means each HCP B must use a tool for IPS visualization compatible with the information model and data structures employed by NCP B. The implementation of the IPS in different information models and enabling support for digital transformation ("two-way") between each two countries is the top of the iceberg, because for shortness here we leave out of scope other issues like information security and confidentiality.

All these problems and more become visible when we attempt to create a model of the business process model pertinent to the primary use case. A business process model allows not only to analyze and understand the process activities, but also evaluate key performance indexes of the process execution through simulation (Shahinyan \& Krastev, 2013). Therefore, the main objective in this paper is to design a business process model employing BPMN and use this model to analyze the implementation of the primary use case in a fully functional web application on an openEHR platform with real- life clinical data. A secondary objective is to summarize the advantages and disadvantages in the proposed business process model and the implementation of the primary use case.

\section{PRIMARY USE CASE IMPLEMENTATION}

A detailed analysis of existing descriptions of the primary use case for cross- border IPS exchange allows to identify the sequence of activities and events that execute each one of the actors involved in that use case. 


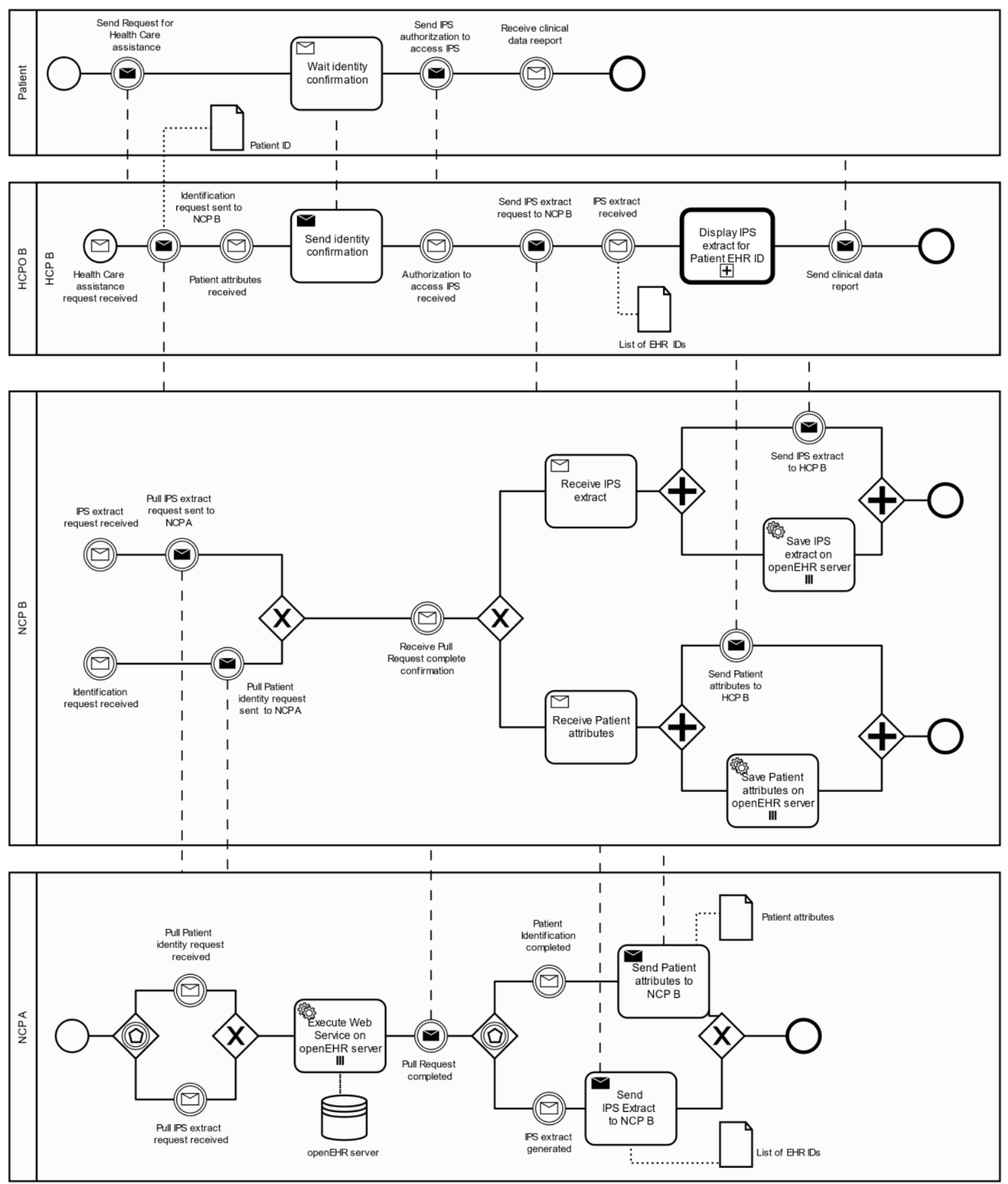

Figure 1. Diagram of the business processes in the Primary Use case of the IPS using BPMN 2.0

Following the syntax requirements in BPMN we design the business process models using open source Business Process Modeler (Camunda, 2020). Figure 1 displays the business processes executed respectively by the Patient, HCP B, NCP B and NCP A. In addition to the visual interpretation each one of these diagrams has XML representation that can be executed on a BPMN Engine for process simulation and evaluation (Rademakers \& van Liempd, 2012).

The business processes on collaborate by means of exchanging message events or executing Send and Receive tasks. The dashed lines between the BPMN swimlanes depict the communication between such events and tasks. 
An important element in the primary use case communications is the communication between NCP B and NCP A. Unlike the description of the flow of events in a UML Use case diagram at this stage of business process modeling we must clarify the type of activity NCP B and NCP A execute. Here we have chosen to use an openEHR server and a stack of web services published by each server to enable the persistence of IPS standard documents and exchange of these documents between NCP B and NCP A. The choice of openEHR platform is not accidental (Pazos, 2020) (openEHR, 2020c). Note activity "Execute Web Services on openEHR Server" executing Service task in the business process owned NCP A. Similar Service tasks are used in the business process owned by NCP B to save the patient attributes and IPS document extracted from NCP A. This choice is motivated by the fact that this platform employs an Archetype information model and this way it satisfies the nonfunctional requirement for semantic interoperability in cross- border IPS exchange. Moreover, openEHR identifies a patient by a unique Electronic Health Record (EHR) ID and this way supports confidentiality and security requirements in accessing sensitive patient data. Hence, in his communication with HCP B the Patient identifies himself with personal attributes, while HCP B receives access to the IPS document through the EHR ID linked to these attributes. In related research (Krastev, et al., 2019) (Krastev, et al., 2020) we have described the representation of an IPS standard document in terms of an openEHR archetype. Once the archetype IPS standard document is shared on public repositories, it will eliminate duplication of efforts and costs to implement IPS standard data structures in heterogenous and usually non- standard information models. Besides, it facilitates smooth and effortless digital sharing of IPS content from NCP A into the openEHR server on NCP B.

Another key contribution in the proposed business model is the introduction of a BPMN Call activity to display the IPS document in the business process of HCP B (see activity "Display IPS extract for EHR ID" in Figure 1). Designed this way the activity is interpreted as a global task in sense that it will be implemented in software once and reused by any other instance of HCP B. Finally, note that at the end of the business process of NCP B makes a copy of the patient attributes and his IPS on the openEHR platform managed by country B. Thus, the IPS copy may be used next time the Patient needs health assistance in country B.

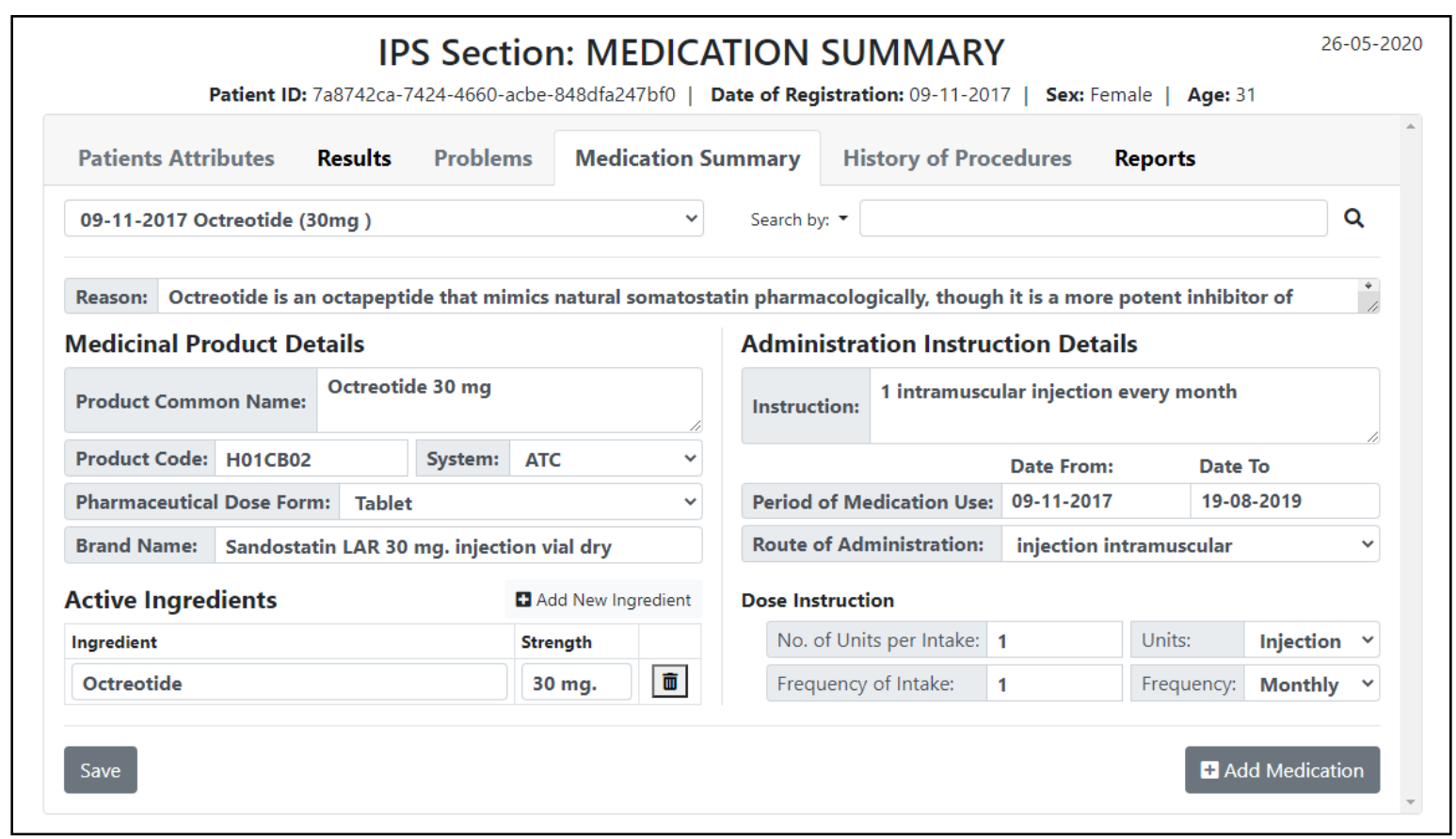

Figure 2. Execution of activity "Display IPS extract for Patient EHR ID" by HCP B

Once the business process model is clarified we attempt to implement it in a real-life web application. To simulate communication between NCP B and NCP A we have created a cluster of two virtual machines running openEHR servers. Additionally, we have developed a PHP client application to execute the business process of HCP B. In this scenario we assume the Patient is a human that collaborates with HCP B in executing validation of patient identity and authorizing the access to the patient's EHR ID. The web 
application is presented in a related research paper (Krastev, et al., 2020). Detailed demonstration in the execution of the web application is provided in (Kovachev, 2020). A sample snapshot of the web page with required Medication summary section of the extracted IPS is shown in Figure 2. The Medication summary section content entirely satisfies the IPS standard, while representing an instance of the openEHR archetype model. For clarity we have preserved in this web page the same label notations and data layout as those used in the IPS standard. This web page allows the HCP B to view previously prescribed medications with detailed semantic context describing each medication. For example, each medication is uniquely identified by its ATC code (WHO Collaborating Centre for Drug Statistics Methodology, 2020), instructions for administering the medication and the active ingredients are part of this description.

\section{DISCUSSION}

The design and the analysis of the business process model for cross- border exchange of IPS documents contributed significantly in identifying missing activities that are required in the software implementation. This model elaborates the existing descriptions of the primary use case. Therefore, its execution is entirely compatible with the functional requirements and the flow of events in that use case description. Moreover, the business process model adds semantic interoperability to the set of non-functional requirements of the originally use case description. Another advantage of this model is the introduction of openEHR as a common platform for providing persistence and web service communication between the actors involved in a real- life scenario (Gutiérrez, 2015). The above presented web application has no analog in the existing literature. It proves that a standard based IPS document can be represented as openEHR archetype. Moreover, instances of this archetype can be used in cross- border exchange (read and write operations) of IPS electronic health records.

Without loss of generality the openEHR platform can be replaced by another platform employing Archetype information model like EN 13606 to represent the IPS clinical document with respect to the existing standard in the European Union. In a related research paper, we have shown the standard IPS document representation in terms of an EN 13606 archetype as well (Krastev, et al., 2019). This approach eliminates duplication of considerable amounts costs and efforts that otherwise are necessary to implement the existing primary use case in practice. The development of archetypes and transformation of existing data into instances of these archetypes is not a straightforward process. It represents one of the disadvantages in implementing the Archetype information model. Employing open source code such as the freely available openEHR platform is acceptable for research purposes (Pazos, 2020). The development of the PHP client of the demonstrated web application needs a lot of programming experience as well. In general, the development of standard- based eHealth applications require a synergy of knowledge in health informatics, healthcare organization and medicine. However, the efforts invested in development of archetypes based on international standards payback and at the end cost less than the implementation of many heterogeneous and incompatible with each other eHealth systems. Moreover, such systems will not be semantically interoperable either.

\section{CONCLUSION}

This paper considers the primary use case for cross- border exchange of clinical documents satisfying the current IPS standard EN 17269. A detailed description of this use case is available in the existing literature, while its implementation in real- life application remains a challenge. This paper presents a novel approach to analyze this use case by designing a BPMN business process model employing a Camunda Modeler. The obtained BPMN model is executable on a BPMN engine and allows to discover missing activities and events of practical importance in the existing scenarios for IPS implementation. For example, scenarios in existing use case descriptions do not take in consideration the activities for transformation between the different IPS information models employed in EU countries. Open source tools are used to design and evaluate the execution of the BPMN process with clinical data. An openEHR platform is introduced in the execution of the process employing the openEHR Archetype information model and adds semantic interoperability capabilities of the software implementation. A sample web application proves the correctness of the obtained 
business process model. The computer experiments show that the web application precisely executes the sequence of activities in the BPMN model. Moreover, the application reads and writes electronic health records of a patient's IPS, whose data structure follows the current EN 17269:2019 standard for this document. The experimental results demonstrate that the electronic health records satisfy both the dual information model of openEHR and the IPS standard EN 17269:2019. We show that the Medication summary section on these records encapsulates detailed semantic context describing each previously prescribed medication.

Both the here developed BPMN model and the web application for cross- border management of IPS records based on the openEHR dual information model and the EN 17269 standard have no analogs in the existing literature. In addition to cross- border exchange of clinical documents this web application can be accommodated locally in each EU country and serve secondary scenarios in FprCEN/TS 17288 comprising both unplanned and planned cases of medical treatment in the healthcare domain. Our current research work is focused on adapting the web application to the requirements of the national legislation for monitoring of patients suffering severe infectious diseases like COVID-19 and prevention the spread of such diseases. In our future work we plan to extend the usability of the web application by implementing the IPS standard EN 17269 in terms of the EN 13606:2019 dual information model.

\section{ACKNOWLEDGEMENT}

This research is supported by the National Scientific Program eHealth in Bulgaria.

\section{REFERENCES}

Camunda, 2020. Modeler. The developer-friendly desktop app for editing BPMN process diagrams and DMN Decision Tables. [Online] Available at: https://camunda.com/products/modeler/ [Accessed 22 May 2020].

CEN/TC 251, 2018. FprCEN/TS 17288. Health informatics - The International Patient Summary: Guidance for European Implementation, Brussels: Eurpoean Committee for Standadization.

CEN/TC 251, 2019a. EN 17269. Health informatics - The Patient Summary for Unscheduled, Cross- border Care, Brussels: Eurpoean Committee for Standadization.

European Commission, 2012. European Patients Smart Open Services (epSOS). D3.2.2 Final definition of functional service requirements- Patient Summary. [Online] Available at: https://ec.europa.eu/digital-singlemarket/en/news/cross-border-health-project-epsos-what-has-it-achieved_[Accessed 5 June 2019].

European Commission, 2015. The Antilope project. [Online] Available at: https://www.antilope-project.eu/ [Accessed 22 May 2020].

European Commission, 2018. eHealth DSI Patient Summary and ePrescription. PS Functional requirements. [Online] Available https://ec.europa.eu/cefdigital/wiki/download/attachments/35210463/PS\%20functional\%20requirements_v2.2.2.pdf [Accessed 20 May 2020].

European Commission, 2020. Use Case Repository.Patient Summary Sharing On A Cross-Border And International Scale. [Online] Available at: https://usecase-repository.ihe-europe.net/content/patient-summary-sharing-cross-borderand-international-scale [Accessed 20 May 2020].

Footman, K. et al., 2014. Policy Summary 14. Cross-border health care in Europe. Copenhagen, Denmark: World Health Organization. Regional Office for Europe. [Online] Available at: https://www.euro.who.int/_data/assets/pdf_file/0009/263538/Cross-border-health-care-in-Europe-Eng.pdf [Accessed 20 June 2020].

Freund, J. \& Rücker, B., 2020. Real-Life BPMN: With introductions to CMMN and DMN. Analyze, improve and automate your business processes. 4 th ed. s.l.:Camunda.

Gutiérrez, P. P., 2015. Towards the Implementation of an openEHR-based Open Source EHR Platform (a vision paper). São Paulo, Brazil, IOS Press, pp. 45-49.

ISO/TC 215, 2019b. ISO 13606-1:2019 Health informatics -- Electronic health record communication -- Part 1: Reference model. [Online] Available at: https://www.iso.org/standard/67868.html_[Accessed 6 June 2019].

Kovachev, P., 2020. openEHR implementation of International Patient Summary based on the Fpr CEN 17269 standard. [Online] Available at: https://www.youtube.com/watch?v=lDReBt9DsAs [Accessed 19 May 2020]. 
Krastev, E. et al., 2019. Software Implementation of the EU Patient Summary with Archetype Concepts. Porto, Portugal, IARIA, pp. 8- 13 .

Krastev, E., Tcharaktchiev, D., Kovatchev, P. \& Abanos, S., 2020. International Patient Summary Standard Based on Archetype Concepts. International Journal On Advances in Life Sciences, 12(1-2), p. (in print).

openEHR, 2020a. The EHR Information Model. [Online] Available at: https://specifications.openehr.org/releases/RM/latest/ehr.html\#_the_ehr_information_model_[Accessed 4 May 2020].

openEHR, 2020b. Archetype Model (AM) Component - latest. [Online] Available at: https://specifications.openehr.org/releases/AM/latest/index [Accessed 22 May 2020].

openEHR, 2020c. EHRServer. Platform. Free to use \& open source. [Online] Available at: https://www.openehr.org/downloads/platform/_Accessed 10 May 2020].

Pazos, P., 2020. Service-oriented openEHR repository for clinical data with composition commit, query and retrieve capabilities. [Online] Available at: https://github.com/ppazos/cabolabs-ehrserver [Accessed 20 May 2020].

Rademakers, T. \& van Liempd, R., 2012. Activiti in Action. s.l.:Manning Publications.

Shahinyan, K. \& Krastev, E., 2013. Extending a BPMN Engine with Evaluation Metrics for KPIs. Sofia, Sofia University Press, pp. 102- 109.

Tcharaktchiev, D. et al., 2020. Cross-border Exchange of Clinical Data using Archetype Concepts Compatible with the International Patient Summary. In: L. Pape-Haugaard, et al. eds. Digital Personalized Health and Medicine. Proceedings of MIE 2020. Geneva: Studies in Health Technology and Informatics, IOS Press, p. (in print).

WHO Collaborating Centre for Drug Statistics Methodology, 2020. Anatomical Therapeutic Chemical code classification system (ATC). [Online] Available at: https://www.whocc.no/atc_ddd_index/ 